\title{
Antimicrobial Resistance: The 'Other' Pandemic!
}

\author{
Based on 9th Dr. I. C. Verma Excellence Award for Young Pediatricians Delivered as Oration on \\ 19th Sept. 2021
}

\author{
Tanu Singhal ${ }^{1}$
}

Received: 6 October 2021 / Accepted: 25 October 2021 / Published online: 22 January 2022

(c) Dr. K C Chaudhuri Foundation 2022

\begin{abstract}
Antimicrobial resistance is projected to kill 10 million people by 2050. The biggest driver of antimicrobial resistance is excessive/unrestricted use of antimicrobials in humans and animals. Antimicrobial resistance is a problem in all types of pathogens including bacteria, mycobacteria, viruses, fungi, and parasites both globally and India and in both adults and children. The areas of greatest concern for India is the epidemic of MDR and XDR tuberculosis and resistance in gram-negative pathogens. The alarming rate of extended spectrum beta lactamase (ESBL) production in Enterobacteriales in both community and health care-associated infections is driving carbapenem use. Rates of carbapenem resistance are now significantly high in health care-associated gram negative pathogens with associated high mortality rates. The key solution to this antimicrobial resistance crisis needs participation of all stakeholders and lies in promoting rational antimicrobial therapy.
\end{abstract}

Keyword Antimicrobial resistance $\cdot$ India $\cdot$ Children $\cdot$ ESBL $\cdot$ Carbapenem resistance

\section{Introduction}

The pandemic of COVID-19, the worst public health crises in over a century occupies our entire mindspace at this time. This article is to remind the readers of the "other" pandemic of antimicrobial resistance (AMR)! Antimicrobial resistant (AR) pathogens are projected to kill 10 million people annually by the year 2050 as against 700,000 per year currently [1]. India is and will be one of the worst affected countries. The drug pipeline is rapidly drying up with few new drugs on the horizon. Hence, it is crucial that we do whatever possible to try and avert/mitigate the AR crises. While the problem of AMR cuts across all microorganisms including bacteria, mycobacteria, viruses, fungi and parasites, the focus here shall be on antibacterial resistance in Indian children.

\section{Mechanisms of AR [2]}

$\mathrm{AR}$ in pathogens can be innate or acquired. Examples of innate resistance are resistance in Klebsiella to ampicillin, enterococcus to cephalosporins, etc. Acquired resistance is

Tanu Singhal

tanusinghal@yahoo.com

1 Department of Pediatrics and Infectious Disease, Kokilaben Dhirubhai Ambani Hospital and Medical Research Institute, Mumbai, Maharashtra 400053, India due to selection pressure exerted by an antimicrobial which wipes out the sensitive strains and leads to selective proliferation of the resistant strains. Mechanisms for antibiotic resistance include production of inactivating enzymes (e.g., beta lactamases, aminoglycoside modifying enzymes), drug efflux (resistance to macrolides, meropenem), inhibition of drug entry (resistance to imipenem), target modification (resistance to methicillin) and alteration of metabolic pathway (resistance to cotrimoxazole). Resistance may be chromosomally or plasmid mediated and multiple mechanisms for resistance to one drug/multiple drugs coexist in the same pathogen.

\section{Why Does Antimicrobial Resistance Occur?}

Antimicrobial overuse is the biggest driver for AMR by exerting selection pressure [3]. Remarkably, while about one third of the total antibiotic use is in humans, two third is in animals primarily for growth promotion [4]. Environment contamination with antimicrobials due to improper handling of human excreta, contaminated effluent from hospitals/ pharmaceutical companies and use of biocides in agriculture also drives AMR [4]. 


\section{What Are the Reasons for Antimicrobial Overuse?}

India is one of the biggest consumer of antibiotic globally with 10.7 units/person annually [5]. Understanding the underlying reasons and the magnitude of the problem is crucial to devise corrective measures. Overuse is due to two main factors [3].

- High burden of infections which in turn is due to low immunization rates, lack of good water and sanitation, poor hygiene, overcrowding, air pollution, malnutrition, other environmental factors that lead to increased prevalence of vector borne diseases and poor infection control in hospitals.

- Irrational/Excessive use of antibiotics which in turn is due to poor prescriber knowledge, pressure from parents/caregivers, over the counter (OTC) availability of antibiotics, low costs of antibiotics, pressure from pharmaceutical companies, fear of medicolegal repercussions for missing a diagnosis and often in order to circumvent/ avoid investigations. Availability of substandard antibiotics lowers treatment efficacy and also contributes to resistance.

A recently published study which looked at outpatient antibiotic prescriptions in the private sector in India through IQVIA reported 519 million annual prescriptions in 2013-2014, with rate of 412 prescriptions/1000 person years (approximately one out of two people) [6]. Notably the rate was highest in children aged $0-4 \mathrm{y}$ and $20 \%$ of the prescriptions were for upper respiratory tract infections and $10 \%$ for other primarily nonbacterial illnesses such as cough, nasopharyngitis, asthma, etc. Hence, this study and other similar ones indicatetremendous scope to reduce antibiotic use in the community.

The pandemic of COVID-19 has further worsened India's antimicrobial crises with unrestricted prescriptions of hydroxychloroquine, azithromycin, doxycycline, ivermectin in the community and excess use of antibiotics in hospitalized patients due to secondary infections $[7,8]$.

\section{Current Burden of Antimicrobial Resistance in Indian Children}

Resistant bacteria can be mono drug resistant, multidrug resistant (MDR), extensively drug resistant (XDR) and pan/ total drug resistant (PDR/TDR) [9]. MDR refers to resistance to at least one antimicrobial agent from three or more classes, XDR refers to resistance to all except drugs in 2 classes, while PDR refers to resistance to all available drugs.

\section{Resistance in Community-Acquired Pathogens}

\section{S. pneumoniae}

Pneumococci are responsible for about $10 \%$ of the one million under 5 childhood deaths annually in India [10]. Pneumococcal resistance to beta lactams is by alteration of penicillin binding protein (PBP). Breakpoints for defining resistance to penicillin and cephalosporins were revised downwards for meningeal isolates and upwards for nonmeningeal isolates in 2008 [11]. Resistance in pneumococci is increasing in India. Singh et al. in a review of 7 studies in children below 5 y (2009-2016) reported the incidence of resistance to penicillin as $10 \%$ and to cefotaxime as $4 \%$ [12]. In the ASIP study in children below $5 \mathrm{y}$ which included 361 isolates, penicillin resistance was $8 \%$ and no cephalosporin resistance was reported [13]. However, the largest retrospective study till date is from CMC, Vellore (861invasive isolates in both adults and children, 2008-2016) [14]. Among the 663 nonmeningeal isolates only 1 was fully resistant to penicillin and 3 showed intermediate resistance. Of the 148 meningeal isolates, the overall penicillin resistance was $43 \%$ (increased from $9.5 \%$ in 2008 to $43 \%$ in 2016) while the overall cephalosporin resistance was $15 \%$ (increased from $4.7 \%$ in 2008 to $28.5 \%$ in 2016). This study triggered the change in ICMR guidelines for empiric treatment of bacterial meningitis to include vancomycin along with ceftriaxone [15].

\section{S. aureus}

Community acquired (CA) S. aureus is responsible for skin, soft tissue, bone and joint infections and pneumonia in children. There is a rising prevalence of methicillin resistance in CA pathogens through PBP alteration. The ICMR AMR report of 2020 [(2251 isolates sent from the outpatient department (OPD of sentinel sites) reports the prevalence of MRSA (methicillin resistant S. aureus) as 38\% (cotrimoxazole and clindamycin resistance rates in the MRSA isolates was $30 \%$ and $35 \%$, respectively)] [16]. The prevalence of MRSA in childhood pyoderma was reported as $20 \%$ in a study from Pondicherry [17]. Nasal colonization by MRSA was reported in $29 \%$ in one study in children from Central India [18]. The rising prevalence of CA MRSA supports the empiric use of anti MRSA drugs in serious cases of SSTI (skin and soft tissue infections), bone and joint infections and necrotizing pneumonia in children.

\section{Salmonella enterica}

It is estimated that there an estimated 2.1-3.4 million cases of typhoid and paratyphoid annually in India and $S$. enterica contributes to $10 \%$ of all fever cases admitted to 
hospital [19]. Prevalence of fluoroquinolones resistance is now more than $90 \%$ [20]. Though there is return of susceptibility to older drugs including ampicillin, cotrimoxazole and chloramphenicol, these drugs are rarely used in treatment due to fear of adverse effects. Commonly used drugs are the third generation cephalosporins (cefixime and ceftriaxone) and azithromycin. Sporadic reports of cephalosporin resistance due to production of a novel ESBL and azithromycin resistance are now being reported in India $[21,22]$. The danger of spread of these XDR strains is a "gathering storm" [23].

\section{Shigella Species}

Shigella causes nearly $5 \%-10 \%$ of all diarrhea in children below $5 \mathrm{y}$ of age in developing countries including India [24]. Apart from resistance to cotrimoxazole and ampicillin, there is high prevalence of resistance to fluoroquinolones in India. Hence antimicrobial choices for bloody diarrhea in India include cefixime and azithromycin. Resistance to cephalosporins is emerging and transfer of this plasmid borne resistance to other enteric pathogens is an area of concern [25].

\section{E. coli}

E. coli is the most common cause of CA urinary tract and intraabdominal infections in children. There is increasing prevalence of ESBL in CA E. coli infections with one report from Chennai reporting $40 \%$ prevalence of ESBL in childhood UTI (urinary tract infections) [26]. Another study from rural central India which studied stool samples collected over 2 y in 125 children aged $1-3$ y ( 7 samples per child and 6 isolates per sample; 4764 isolates) reported fecal $E$. coli resistance rates of $100 \%$ to ampicillin, $40 \%$ to meropenem, $4 \%$ to colistin per child, ESBL rates of $99 \%$ and MDR rates of $100 \%$ (if we consider the child over the entire study period) [27]. The rising resistance in $E$. coli has resulted in increasing use of BL-BLI (beta lactam-beta lactamase inhibitor) combinations/carbapenems in critically sick children with CA UTI/intra-abdominal sepsis.

\section{Neonatal Sepsis}

AR in neonatal sepsis is a cause of serious concern. A community based study on neonatal sepsis from South Asia (2011-2014, which included rural sites in Odisha and Vellore) reported susceptibility to ampicillin and or gentamicin ranging from $50 \%-100 \%$ [28]. In sharp contrast, two large hospital based studies from New Delhi (inborn and out born neonates respectively, 1400 isolates together) reported very high resistance rates $[29,30]$. The commonest pathogens were gram negative (Acinetobacter,
Klebsiella, E. coli the top three) with ESBL, MDR and carbapenem rates varying between 15 and $82 \%$ (lowest in E. coli and highest in Acinetobacter). Candida contributed to almost $25 \%$ of all isolates in the out born neonate study (even in term infants and those with birth weight $>1.5 \mathrm{~kg}$ ) possibly reflective of high rates of antibiotic use in the community.

\section{Health Care-Associated Pathogens}

HAIs (Health-Care associated infections) are usually seen in children in the critical care unit, children undergoing cancer chemotherapy, following surgery and other invasive procedures. The predominant pathogens in the Indian setting are gram negative followed by gram positive and Candida [16].

\section{Gram-Negative Pathogens}

The usual gram negative microbes causing HAI are Klebsiella, E. coli, Acinetobacter and Pseudomonas [16]. Studies have shown ESBL rates excess of $80 \%$ in these pathogens [31]. Most of these ESBL isolates are MDR as they are often co resistant to quinolones, aminoglycosides and other classes. The high MDR rates have resulted in increased use of carbapenems which in turn has stemmed carbapenem resistance and XDR [32]. Most of the carbapenem resistance is mediated by carbapenemases and less often by efflux and porin mechanisms. The most common carbapenemases in the Indian setting are NDM and OXA unlike Europe and USA where KPC predominate [33]. The NDM producing isolates are characterized by high meropenem MICs (minimum inhibitory concentrations) and ineffectiveness of new BL-BLI inhibitors such as ceftazidime avibactam. Besides, many of the salvage drugs for XDR infections such as tigecycline and minocycline are not approved in children. Spiralling carbapenem resistance is driving excess use of polymyxins resulting in emerging polymyxin resistance [34].

The recently published report from ICMR reports extremely high resistance rates from sentinel ICUs in both adults and children in 2020 (Fig. 1) [16]. At the author's institution, resistance rates in children were lower than adults in 2020 (resistance to BL-BLI (beta lactam-beta lactamase inhibitors) $40 \%$ versus $80 \%$ in adults, resistance to carbapenems of $20 \%$ versus $60 \%$ in adults, colistin resistance $0 \%$ versus $20 \%$ in adults) (unpublished data). Resistance rates in HAI therefore vary widely and each unit should generate its own antibiogram to guide empiric therapy of HAI.

\section{Gram-Positive Pathogens}

ICMR reports $50 \%$ of nosocomial $S$. aureus as methicillin resistant in the year 2020 [16]. Few centers are now reporting 


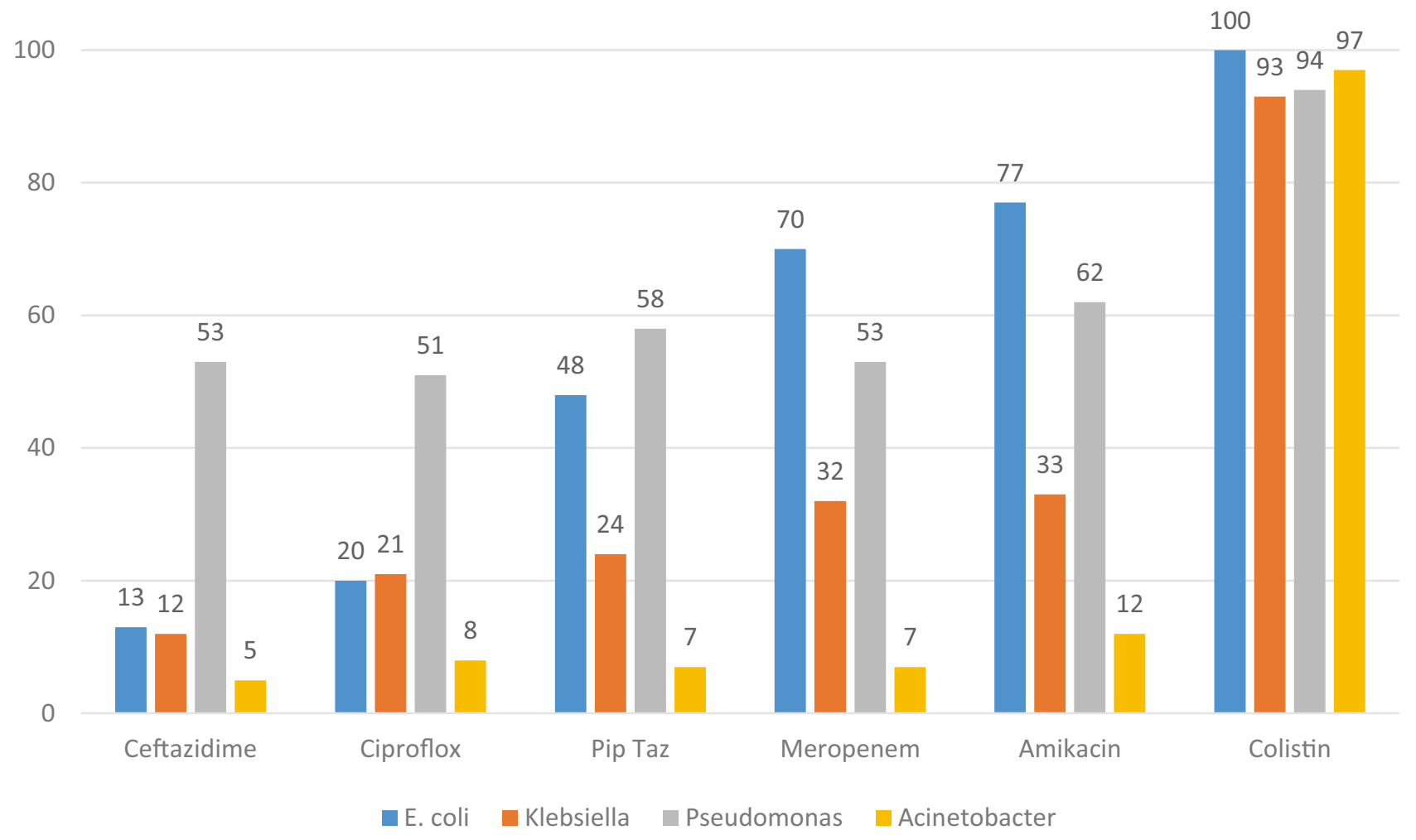

Fig. 1 Antimicrobial susceptibility of ICU pathogens in adults and children, ICMR 2020 report [16]

high vancomycin MICs of $2 \mu \mathrm{g} / \mathrm{mL}$ (though classified as sensitive, this MIC is often associated with failure of therapy) [35]. The prevalence of vancomycin intermediate $S$. aureus (VISA) and vancomycin resistant $S$. aureus (VRSA) is low in India. Linezolid resistance is being sporadically reported in $S$. aureus due to widespread misuse [16]. Coagulase negative staphylococci (CONS) are mainly implicated in blood stream infections in NICU and PICU and in CSF shunt infections; more than $80 \%$ of nosocomial CONS isolates are methicillin resistant [16]. Enterococci are emerging nosocomial pathogens causing blood stream infections with increasing prevalence of vancomycin resistance [16].

\section{Solutions to the Problem of Antimicrobial Resistance}

The solution to the problem of antimicrobial resistance is complex and involves prevention of infections (which will indirectly reduce antibiotic use), tracking of AMR (currently being effectively done by ICMR), promotion of rational antimicrobial therapy and development of new drugs [3].

\section{Prevent Infections}

This strategy apart from reducing AMR will also reduce childhood morbidity and mortality. The key interventions here are improving immunization services and provision of safe water, improving sanitation, reducing environmental pollution, promotion of hand hygiene, breast feeding and improving nutrition. While pneumococcal and rotavirus vaccines are being introduced in the national program in a staggered manner, introduction of typhoid vaccines is also a priority [36].

Hospital Infection Prevention and Control is another key intervention area. Good hospital infection control practices reduce the occurrence of infections due to MDR/ XDR pathogens and thus favorably impact resistance rates to high end antibiotics. All neonatal and pediatric ICUs (intensive care unit) must have a comprehensive infection control program which should include the following [37].

- Hand hygiene policy

- Prevention bundles for catheter related blood stream infections (CLABSI), health care associated pneumonia 
Fig. 2 Schematic representation of some interventions to promote rational antimicrobial therapy

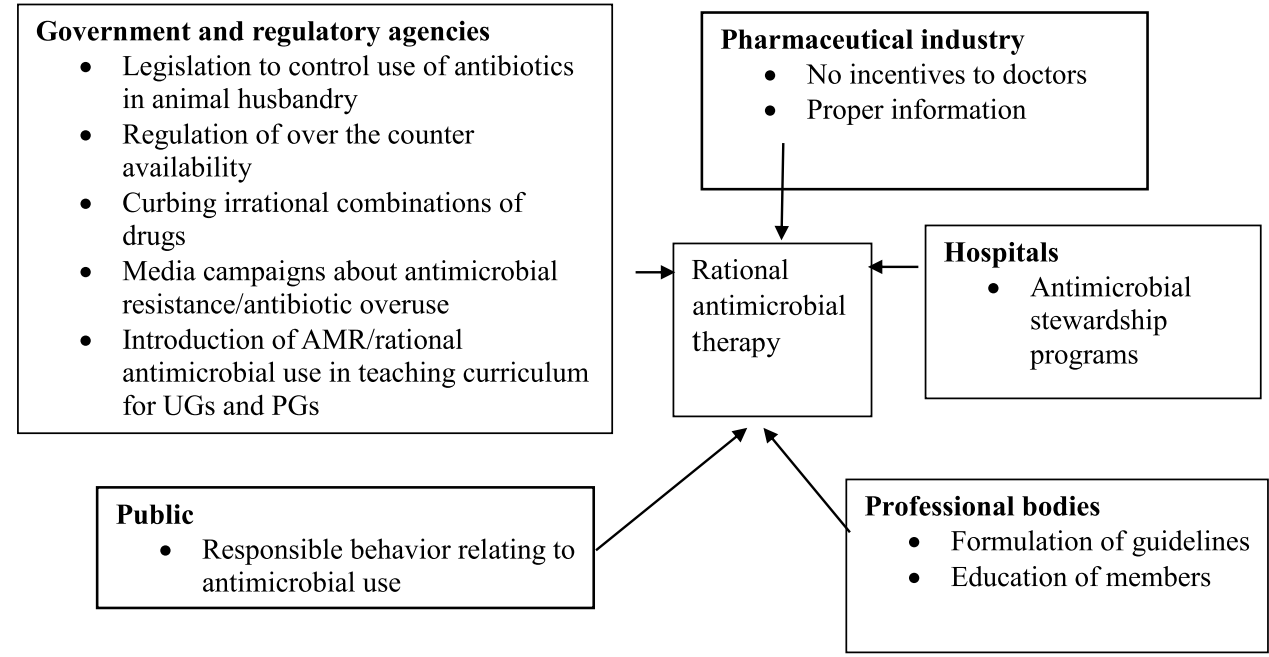

(HAP), catheter related urinary tract infections (CAUTI) and surgical site infections (SSI).

- Policies for environmental infection control (air, water and surfaces).

- Isolation policies including contact isolation for patients colonized/ infected with carbapenem-resistant pathogens, MRSA, VRE (vancomycin resistant enterococci).

- Tracking of sentinel infection rates including CLABSI, CAUTI, HAP, and SSI rates and comparison with benchmarks.

- Mechanism for real time detection and management of outbreaks [38].

Active involvement of the neonatologist/pediatric critical care specialist with the clinical microbiologist and nursing is integral to drive a good infection control program.

\section{Promotion of Rational Antimicrobial Therapy (Fig. 2)}

This involves all stakeholders including the government, pharmaceutical industry, professional bodies, health care professionals, media and the public [39, 40].

The role of the government and regulatory agencies involves appropriate legislation to control use of antibiotics in animal industry (colistin use in animal feed was recently banned), regulation of OTC availability of antibiotics (some steps are being taken in this direction), curbing irrational combinations of drugsand substandard generic drugs (this needs more attention), promotion of media campaigns about antimicrobial resistance and dangers of antibiotic overuse (akin to campaigns about tuberculosis and COVID-19), introduction of AMR/rational antimicrobial use in teaching curriculum for undergraduates and post graduates etc. Linking renewal of medical licenses to training in antimicrobial therapy will help upgrade knowledge of practicing medical professionals. Making cheap and point of care diagnostics available will also rationalize antimicrobial therapy by facilitating accurate diagnosis. Formulation of standard treatment guidelines to manage infections and dissemination of the same to the medical community is crucial (one such guideline was recently published by ICMR) [15].

Professional bodies have a key role to play in educating their members about rational antimicrobial therapy. The Indian Academy of Pediatrics has taken several steps in this direction in the past decade with development of teaching modules, formulation of guidelines and publication of books [41]. The public should also behave responsibly and avoid self-medication and OTC use of antibiotics. The pharmaceutical industry should practice restraint while promoting their portfolio, avoid giving incentives to doctors for antibiotic prescriptions and reinforce the principle of stewardship while interacting with doctors.

While all the above mentioned measures cut down antibiotic abuse in the community, a hospital based antimicrobial stewardship programs (AMSP) is integral to cut down antibiotic misuse in the hospital setting and is discussed further.

\section{Hospital-Based Antimicrobial Stewardship Program (AMSP)}

Setting up of AMSP is now considered a pre requisite for hospital accreditation. Comprehensive guidelines for setting up AMSP have been published by ICMR and Infectious Disease Society of America [42, 43]. Guidelines for setting up a pediatric AMSP have also been published [44]. The antimicrobial stewardship team is usually led by the clinical microbiologist and an infectious disease specialist or a physician/pediatrician with interest in infectious disease. The other members of the team are clinical pharmacist/ pharmacologist, infection control nurses, quality managers and a representative from the hospital IT system. Other supporting members of the AMS committee include surgeons, 
critical care physicians, emergency physicians and hemato oncologists. The core team should be made accountable and reimbursed for time spent in stewardship activities to ensure continuity and effectiveness of the program.

The next step is formulation of guidelines for various infections which includes those for community based infections, surgical prophylaxis and health care associated infections. The guidelines for HAI should be based on the local antibiogram and updated regularly. All treatment guidelines should include culture methods, choice of empiric therapy, modification of further therapy, escalation and de-escalation and dose and duration of antimicrobials. Once the guidelines have been developed they should be disseminated among the stakeholders through seminars, posters, booklets and even smart phone based applications. Knowledge of the AMS program should be tested on a regular basis by quizzes and games with prizes.

Audit of antimicrobial use is an integral part of the program. There are two such strategies; one is front end strategy in which prescription of a high end antibiotic requires clearance from the clinical microbiologist/infectious disease specialist. This is however not the preferred strategy since it may not be possible to withhold high-end antibiotics from a very sick patient and has found poor acceptance. Therefore, the strategy most commonly adopted is a back end strategy. Here the use of certain high end antibiotics (egpolymyxins, anti MRSA drugs, antifungals) is audited after $48-72 \mathrm{~h}$ of prescription by a stewardship committee. The committee decides on the justification of the antibiotic based on the clinical details, culture results and response to therapy. This decision is communicated to the prescriber and a consensual decision is taken to continue or stop the antibiotic. The acceptance of the back end strategy is better though it is more labor intensive [45].

Other methods that help in antimicrobial stewardship include selective reporting/cascading of reports by microbiology lab. Here if the isolated pathogen is sensitive to low end antibiotics, susceptibility to high end drugs is not reported. Resource intensive methods including multiplex PCR for identification of viral pathogens and MALDI TOF (matrix assisted laser desorption ionization time of flight analysis) for quick identification of blood stream isolates also helps in stewardship. Use of biomarkers (beta D glucan, galactomannan) help to control antifungal therapy. Use of procalcitonin based algorithms assist in cutting down antibiotic duration. Pharmacy based interventions that allow for early IV to oral switch, dosing, checking for interactions and pharmacodynamic dose optimization are also useful.

The effectiveness of any AMSP has to be evaluated by appropriate metrics. These include antibiotic measures such as antimicrobial measures [antimicrobial defined daily dose (DDD); antimicrobial days of therapy (DOT) length of therapy (LOT)]. Microbial measures include prevalence of antimicrobial resistant organisms and prevalence/incidence of $C$. difficile. Clinical outcomes that evaluate the effectiveness include mortality, length of stay, and cure.

It is also recommended that all pediatric and neonatal units set up an internal stewardship program to control antibiotic use in addition to the one set up by the hospital.

\section{Conclusions}

We are facing an "antimicrobial resistance" crises. We are running out of treatment options and not many new drugs are in the pipeline. If we do not use our existing antimicrobials wisely we are destined to return to the pre-antibiotic era of untreatable infections.

Acknowledgements The author acknowledges the contribution of Dr SK Kabra, Late Dr MK Bhan, Dr Camilla Rodrigues, Dr Rajeev Soman, Late Dr Ajita Mehta, Dr Suhas Prabhu, Dr Sweta Shah, Dr Vatsal Kothari, Dr Pooja Thakkar and Sister Reshma Naik.

\section{Declarations}

Conflict of Interest None.

\section{References}

1. O'Neill J. Review on Antimicrobial Resistance. Antimicrobial Resistance: Tackling a crisis for the health and wealth of nations. London: Review on Antimicrobial Resistance. 2014. Available at: https:// amr-review.org/sites/default/files/AMR\%20Review\%20Paper\% 20-\%20Tackling $\% 20$ a\%20crisis $\% 20$ for $\% 20$ the $\% 20$ health $\% 20$ and $\%$ 20wealth\%20of\%20nations_1.pdf. Accessed on 30th Sept 2021.

2. Kaye KS, Engemann JJ, Fraimow HS, Abrutyn E. Pathogens resistant to antimicrobial agents: epidemiology, molecular mechanisms, and clinical management. Infect Dis Clin North Am. 2004;18:467-511.

3. Laxminarayan R, Chaudhury RR. Antibiotic Resistance in India: drivers and opportunities for action. PLoS Med. 2016;13:e1001974.

4. Taneja N, Sharma M. Antimicrobial resistance in the environment: The Indian scenario. Indian J Med Res. 2019;149:119-28.

5. Van Boeckel TP, Gandra S, Ashok A, et al. Global antibiotic consumption 2000 to 2010: an analysis of national pharmaceutical sales data. Lancet Infect Dis. 2014;14:742-50.

6. Farooqui HH, Mehta A, Selvaraj S. Outpatient antibiotic prescription rate and pattern in the private sector in India: Evidence from medical audit data. PLoS One. 2019;14:e0224848.

7. Sulis G, Batomen B, Kotwani A, Pai M, Gandra S. Sales of antibiotics and hydroxychloroquine in India during the COVID19 epidemic: an interrupted time series analysis. PLoS Med. 2021;18:e1003682.

8. Khurana S, Singh P, Sharad N, et al. Profile of co-infections $\&$ secondary infections in COVID-19 patients at a dedicated COVID-19 facility of a tertiary care Indian hospital: implication on antimicrobial resistance. Indian J Med Microbiol. 2021;39:147-53.

9. Magiorakos AP, Srinivasan A, Carey RB, et al. Multidrug-resistant, extensively drug-resistant and pandrug-esistant bacteria: an international expert proposal for interim standard definitions for acquired resistance. Clin Microbiol Infect. 2012;18:268-81. 
10. Farooqui H, Jit M, Heymann DL, Zodpey S. Burden of severe pneumonia, pneumococcal pneumonia and pneumonia deaths in Indian states: modelling based estimates. PLoS One. 2015;10:e0129191.

11. Clinical and Laboratory Standards Institute. M100 Performance standards for antimicrobial susceptibility testing; 31 st Edition. Clinical and Laboratory Standards Institute; Wayne, PA. 2021. p.92-95.

12. Singh J, Sundaresan S, Manoharan A, Shet A. Serotype distribution and antimicrobial susceptibility pattern in children $\leq 5$ years with invasive pneumococcal disease in India - a systematic review. Vaccine. 2017;35:4501-9.

13. Manoharan A, Manchanda V, Balasubramanian S, et al. Alliance for Surveillance of Invasive Pneumococci (ASIP) Study Group. Invasive pneumococcal disease in children aged younger than 5 years in India: a surveillance study. Lancet Infect Dis. 2017;17:305-12.

14. Verghese VP, Veeraraghavan B, Jayaraman R, et al. Increasing incidence of penicillin- and cefotaxime-resistant streptococcus pneumoniae causing meningitis in India: time for revision of treatment guidelines? Indian J Med Microbiol. 2017;35:228-36.

15. Indian Council for Medical Research. Treatment Guidelines for Antimicrobial Use in Common Syndromes. 2019. Available at https://main.icmr.nic.in/sites/default/files/guidelines/Treatment Guidelines_2019_Final.pdf. Accessed on 1st Oct 2021.

16. Indian Council for Medical Research. Antimicrobial Resistance Research and Surveillance Network. 2020. Available at: https:// main.icmr.nic.in/sites/default/files/guidelines/AMRSN_annual_ report_2020.pdf . Accessed on 1st Oct 2021.

17. Venniyil PV, Ganguly S, Kuruvila S, Devi S. A study of communityassociated methicillin-resistant Staphylococcus aureus in patients with pyoderma. Indian Dermatol Online J. 2016;7:159-63.

18. Dey S, Rosales-Klintz S, Shouche S, Pathak JP, Pathak A. Prevalence and risk factors for nasal carriage of Staphylococcus aureus in children attending anganwaries (preschools) in Ujjain. India BMC Res Notes. 2013;6:265.

19. John J, Van Aart CJ, Grassly NC. The burden of typhoid and paratyphoid in India: systematic review and meta-analysis. PLoS Negl Trop Dis. 2016;10:e004616.

20. Britto CD, John J, Verghese VP, Pollard AJ. A systematic review of antimicrobial resistance of typhoidal Salmonella in India. Indian J Med Res. 2019;149:151-63.

21. Jacob JJ, Pragasam AK, Vasudevan K, et al. Salmonella Typhi acquires diverse plasmids from other Enterobacteriaceae to develop cephalosporin resistance. Genomics. 2021;113:2171-6.

22. Carey ME, Jain R, Yousuf M, al. Spontaneous emergence of azithromycin resistance in independent lineages of Salmonella Typhi in Northern India. Clin Infect Dis. 2021;72:e120-7.

23. Levine MM, Simon R. The gathering storm: is untreatable typhoid fever on the way? mBio. 2018;9:e00482-18.

24. Taneja N, Mewara A. Shigellosis. epidemiology in India. Indian J Med Res. 2016;143:565-76.

25. Sethuvel DPM, Perumalla S, Anandan S, et al. Antimicrobial resistance, virulence \& plasmid profiles among clinical isolates of Shigella serogroups. Indian J Med Res. 2019;149:247-56.

26. Balasubramanian S, Kuppuswamy D, Padmanabhan S, Chandramohan V, Amperayani S. Extended-spectrum Beta-lactamase-producing Community-acquired urinary tract infections in children: chart review of risk factors. J Glob Infect Dis. 2018;10:222-5.

27. Purohit MR, Lindahl LF, Diwan V, Marrone G, Lundborg CS. High levels of drug resistance in commensal E. coli in a cohort of children from rural central India. Sci Rep. 2019;9:6682.

28. Saha SK, Schrag SJ, El Arifeen S, et al. Causes and incidence of community-acquired serious infections among young children in south Asia (ANISA): an observational cohort study. Lancet. 2018;392:145-59.

29. Investigators of the Delhi Neonatal Infection Study (DeNIS) collaboration. Characterisation and antimicrobial resistance of sepsis pathogens in neonates born in tertiary care centres in Delhi, India: a cohort study. Lancet Glob Health. 2016;4:e752-60.

30. Jajoo M, Manchanda V, Chaurasia S, et al. Investigators of the Delhi Neonatal Infection Study (DeNIS) collaboration, New Delhi, India. Alarming rates of antimicrobial resistance and fungal sepsis in outborn neonates in North India. PLoS One. 2018;13:e0180705.

31. Singhal T, Shah S, Thakkar P, Naik R. The incidence, aetiology and antimicrobial susceptibility of central line-associated bloodstream infections in intensive care unit patients at a private tertiary care hospital in Mumbai. India Indian J Med Microbiol. 2019;37:521-6.

32. Alagesan M, Gopalakrishnan R, Panchatcharam SN, Dorairajan S, MandayamAnanth T, Venkatasubramanian R. A decade of change in susceptibility patterns of Gram-negative blood culture isolates: a single centre study. Germs. 2015;5:65-77.

33. Veeraraghavan B, Pragasam AK, Bakthavatchalam YD, Anandan S, Swaminathan S, Sundaram B. Colistin-sparing approaches with newer antimicrobials to treat carbapenem-resistant organisms: Current evidence and future prospects. Indian J Med Microbiol. 2019;37:72-90.

34. Banerjee T, Wangkheimayum J, Sharma S, Kumar A, Bhattacharjee A. Extensively drug-resistant hypervirulent Klebsiella pneumoniae From a series of neonatal sepsis in a tertiary care hospital, India. Front Med (Lausanne). 2021;8:645955.

35. Mohanty S, Behera B, Sahu S, Praharaj AK. Recent pattern of antibiotic resistance in Staphylococcus aureus clinical isolates in Eastern India and the emergence of reduced susceptibility to vancomycin. J Lab Physicians. 2019;11:340-5.

36. Dash N, Rose W. Typhoid conjugate vaccine: is it time for it to be in the national immunization schedule? Indian Pediatr. 2020;57:609-10.

37. Indian Council of Medical Research. Hospital Infection Control Guidelines. 2017. Available at: https://main.icmr.nic.in/sites/ default/files/guidelines/Hospital_Infection_control_guidelines. pdf. Accessed on 25th Sep 2021.

38. Paul LM, Hegde A, Pai T, Shetty S, Baliga S, Shenoy S. An outbreak of Burkholderia cepacia bacteremia in a neonatal intensive care unit. Indian J Pediatr. 2016;83:285-8.

39. Ghafur A, Mathai D, Muruganathan A, et al. The Chennai Declaration: a roadmap to tackle the challenge of antimicrobial resistance. Indian J Cancer. 2013;50:71-3.

40. Singh S, Charani E, Devi S, et al. A road-map for addressing antimicrobial resistance in low- and middle-income countries: lessons learnt from the public private participation and co-designed antimicrobial stewardship programme in the State of Kerala. India Antimicrob Resist Infect Control. 2021;10:32.

41. Yewale VN. IAP-ICMR call to action to tackle the antimicrobial resistance. Indian Pediatr. 2014;51:437-9.

42. Indian Council of Medical Research. Antimicrobial Stewardship Program Guideline. 2018. Available at https://main.icmr.nic.in/sites/ default/files/guidelines/AMSP_0.pdf. Accessed on 30th Sep 2021.

43. Barlam TF, Cosgrove SE, Abbo LM, et al. Implementing an antibiotic stewardship program: guidelines by the infectious diseases society of America and the society for healthcare epidemiology of America. Clin Infect Dis. 2016;62:e51-77.

44. Probst V, Islamovic F, Mirza A. Antimicrobial stewardship program in pediatric medicine. Pediatr Investig. 2021;5:229-38.

45. Thakkar P, Singhal T, Shah S, et al. The implementation and outcome of a 2-year prospective audit and feedback based antimicrobial stewardship program at a private tertiary care hospital. Indian J Med Microbiol. 2021. https://doi.org/10.1016/j.ijmmb.2021.09.005.

Publisher's Note Springer Nature remains neutral with regard to jurisdictional claims in published maps and institutional affiliations. 